\title{
COEFFICIENT, DISTORTION AND GROWTH INEQUALITIES FOR CERTAIN $p$-VALENT CLOSE-TO-CONVEX FUNCTIONS
}

\section{I.B. BAPANA AND SNEHA NANDWANA}

\begin{abstract}
In the present paper, we introduce and investigate a new subclass $\chi_{p}^{t}(\gamma)$ of analytic and $p$-valent close-to-convex functions in the open unit disk $U$. For functions belonging to the class $\chi_{p}^{t}(\gamma)$, some interesting properties including the coefficient estimates, distortion theorems and subordination results are obtained.
\end{abstract}

Mathematics Subject Classification 2000: 30C45

Additional Key Words and Phrases: Analytic p-valent functions, Starlike functions, Closeto-convex functions, Hadamard product, Subordination.

\section{Introduction}

Let $\mathcal{A}(p)$ be the class of functions of the form

$$
f(z)=z^{p}+\sum_{n=1}^{\infty} a_{n+p} z^{n+p} \quad(p \in \mathbb{N}=\{1,2,3, \ldots\}),
$$

which are analytic and $p$-valent in the unit disc $\mathbb{U}=\{z: z \in C,|z|<1\}$. Let $\mathcal{S}^{*}, \quad\left[\mathcal{S}_{p}^{*}(\alpha)\left[\right.\right.$ and $\mathcal{K}_{p}(\alpha)$ be the usual class of functions which are univalent starlike of order zero, $p$-valent starlike and $p$-valent convex of order $\alpha$ where $(0 \leq \alpha<p)$.

For two functions $f$ and $g$, analytic in $\mathbb{U}$, we say that the function $f(z)$ is subordinate to $g(z)$ in $\mathbb{U}$, and we write $f(z) \prec g(z)(z \in \mathbb{U})$, if there exists a Schwartz function $w(z)$, analytic in $\mathbb{U}$ with $w(0)=0$ and $|w(z)|<1$, such that $f(z)=g(w(z)),(z \in \mathbb{U})$. Indeed it is known that

$$
f(z) \prec g(z)(z \in \mathbb{U}) \Leftrightarrow f(0)=g(0) \text { and } f(\mathbb{U}) \subset g(\mathbb{U}) \text {. }
$$

More recently, Kowalczyk et. al [2] discussed $\mathcal{K}_{s}(\gamma)$ of analytic functions related to starlike function.

A function $f(z) \in \mathbb{U}$ is said to be in the class $\mathcal{K}_{s}(\gamma), 0 \leq \gamma<1$, if it satisfies the inequality

$$
\operatorname{Re}\left(\frac{-z^{2} f^{\prime}(z)}{g(z) g(-z)}\right)>\gamma
$$

where $g(z) \in \mathcal{S}^{*}\left(\frac{1}{2}\right)$.

Motivated by this class $\mathcal{K}_{s}(\gamma)$, we introduce a new class $\chi_{p}^{t}(\gamma)(|t| \leq 1, t \neq 0,0 \leq$ $\gamma<p)$.

Definition 1. Let $f$ be a $p$-valent analytic function in $\mathbb{U}$ defined by (1.1) then $f$ 
is said to be in the class $\chi_{p}^{t}(\gamma)(|t| \leq 1, t \neq 0,0 \leq \gamma<p)$ if there exist a function $g \in S^{*}\left(\frac{p}{2}\right)$, such that

$$
\operatorname{Re}\left(\frac{t^{p} z^{p+1} f^{\prime}(z)}{g(z) g(t z)}\right)>\gamma \quad z \in \mathbb{U}
$$

we see that $\chi_{1}^{-1}(\gamma)=K_{s}(\gamma)$ and $\chi_{1}^{-1}(0)=K_{s}$ and by simple calculation we see that

$$
\left|\frac{t^{p} z^{p+1} f^{\prime}(z)}{g(z) g(t z)}+p\right|<\left|\frac{t^{p} z^{p+1} f^{\prime}(z)}{g(z) g(t z)}-p+2 \gamma\right| \quad z \in U
$$

Cho and Zhou [2] and Knwalczyk and Le's-Bomba [3], have obtained properties for the function classes $\mathcal{K}_{s}$ and $\mathcal{K}_{s}(\gamma)$, respectively. Moreover, some other interesting results on the classes $\mathcal{K}_{s}$ and $\mathcal{K}_{s}(\gamma)$ were obtained in $[4,5]$. In the present paper we investigate sufficient conditions, coefficient inequalities and distortion theorems for belonging to th the class $\chi_{p}^{t}(\gamma)$.

\section{Main Results}

Theorem 2.1 Let $g \in S^{*}\left(\frac{p}{2}\right)$ and be given by

$$
g(z)=z^{p}+\sum_{n=1}^{\infty} b_{n+p} z^{n+p}, \quad(z \in U)
$$

If we put

$$
F(z)=\frac{g(z) g(t z)}{t^{p} z^{p}}=z^{p}+\sum_{n=1}^{\infty} c_{p+n} z^{p+n}
$$

where for $n=1,2, \ldots$

$$
c_{p+n}=b_{p+n}+b_{2} b_{p+n-1} t+b_{3} b_{p+n-2} t^{2}+\ldots+b_{p+n-1} b_{2} t^{p+n-2}+b_{p+n} t^{p+n-1}
$$

Then $F \in S^{*}$

Proof. Result (2.2) can be found easily. Also $|t z|<|z|<1$, then from the definition of starlike function we have

$$
\operatorname{Re}\left(\frac{z g^{\prime}(z)}{g(z)}\right)>\frac{p}{2} \text { and } \operatorname{Re}\left(\frac{t z g^{\prime}(t z)}{g(t z)}\right)>\frac{p}{2}
$$

therefore

$$
\begin{gathered}
\operatorname{Re}\left(\frac{z F^{\prime}(z)}{F(z)}\right)=\operatorname{Re}\left(\frac{z g^{\prime}(z)}{g(z)}\right)+\operatorname{Re}\left(\frac{t z g^{\prime}(t z)}{g(t z)}\right)-p \\
\operatorname{Re}\left(\frac{z F^{\prime}(z)}{F(z)}\right)>0
\end{gathered}
$$

hence $F(z) \in S^{*}$, the theorem proved.

Theorem 2.2 A function $f(z) \in \chi_{p}^{t}(\gamma)(0 \leq \gamma<p)$ if and only if there exist $g \in S^{*}\left(\frac{p}{2}\right)$ such that

$$
\frac{t^{p} z^{p+1} f^{\prime}(z)}{g(z) g(t z)} \prec \frac{1+(p-2 \gamma)}{1-z} \quad z \in \mathbb{U}
$$


Proof Let $f(z) \in \chi_{p}^{t}(\gamma)$. then, there exist a function $g(z) \in S^{*}\left(\frac{p}{2}\right)$ such that

$$
\operatorname{Re}\left(\frac{t^{p} z^{p+1} f^{\prime}(z)}{g(z) g(t z)}\right)>\gamma \quad z \in \mathbb{U}
$$

in terms of subordination we can write that

$$
\frac{t^{p} z^{p+1} f^{\prime}(z)}{g(z) g(t z)} \prec \frac{1+(p-2 \gamma) z}{1-z}
$$

because the function $h(z)=\frac{1+(p-2 \gamma)}{1-z}$ is analytic in $\mathbb{U}$ and $h(0)=H(0)=1$, where $H(z)=\frac{t^{p} z^{p+1} f^{\prime}(z)}{g(z) g(t z)}$. Conversely we assume that the subordination (2.4) holds, then there exist an analytic function in $w$ in $\mathbb{U}$ such that $w(0)=0,|w(z)|<1$ and

$$
\frac{t^{p} z^{p+1} f^{\prime}(z)}{g(z) g(t z)} \prec \frac{1+(p-2 \gamma) w(z)}{1-w(z)}
$$

therefore, by using the condition $|w(z)|<1$, we obtain (1.3) which is equivalent to (1.2) so $f(z) \in \chi_{p}^{t}(\gamma)$.

Theorem 2.3 For $0 \leq \gamma<p$, if there function $f(z) \in \chi_{p}^{t}(\gamma)$, then

$$
(p+n)\left|a_{p+n}\right| \leq\left|c_{p+n}\right|+2(p-\gamma)\left(1+\sum_{k=1}^{p+n-1}\left|c_{k}\right|\right)
$$

Proof. Let

$$
p(z)=\frac{1}{p-\gamma}\left(\frac{z f^{\prime}(z)}{F(z)}-\gamma\right)
$$

or

$$
z f^{\prime}(z)=[1+(p-\gamma)(h(z)-1)] F(z)
$$

also

$$
h(z)=1+d_{1} z+d_{2} z^{2}+\ldots
$$

where now $\operatorname{Re}(h(z))>0$ using $(2.2),(2.8)$, in (2.7)

$$
(p+n) a_{p+n}=(p-\gamma)\left(d_{n-1}+d_{n-2} c_{p+1}+\ldots+d_{1} c_{p+n}\right)
$$

since $\operatorname{Re}(p(z))>0$, then $\left|d_{n}\right| \leq 2, n \in N$. Using this property and principle of mathematical induction we obtain

$$
(p+n)\left|a_{p+n}\right| \leq\left|c_{p+n}\right|+2(p-\gamma)\left(1+\sum_{n=1}^{\infty}\left|c_{p+n}\right|\right)
$$

this completes the proof of the theorem 2.2.

Corollary 2.1 Let $0 \leq \gamma<p$ if the function $f \in \chi_{p}^{t}(\gamma)$

$$
\left|a_{p+n}\right| \leq 1+(p+n-1)(p-\gamma)
$$

Proof. From theorem (2.1) $F(z) \in S^{*}$, thus $\left|c_{p+n}\right| \leq p+n$ The assertion (2.9), can now easily derived from theorem $(2.3)$.

Remark. Setting $p=1, t=-1$ in theorem 2.2 we get the reuslts due to Geo and 
Zhou [2]

Theorem 2.4 Let $g \in s^{*}\left(\frac{p}{2}\right)$ be a function given by (1.2), and $0 \leq \gamma \leq p$. If an analytic function $f$ in $\mathbb{U}$ defined by (1.1) satisfies the inequality

$$
2 \sum(n+p)\left|a_{n+p}\right|+(|p-2 \gamma|+p) \sum_{n=1}^{\infty}\left|c_{n+p}\right| \leq 2(p-\gamma)
$$

where $\mathrm{n}=1,2 \ldots$ and the coefficient $c_{n+p}$ are given by $(2.3)$, then $f \in \chi_{p}^{t}(\gamma)$.

Proof Let

$$
\begin{aligned}
\Lambda= & \left|z f^{\prime}(z)-p \frac{g(z) g(t z)}{z^{p} t^{p}}\right|-\left|z f^{\prime}(z)-\frac{(p-2 \gamma) g(z) g(t z)}{z^{p} t^{p}}\right| \\
& =\left|\sum_{n=1}^{\infty}(n+p) a_{n+p} z^{n+p}-p \sum_{n=1}^{\infty} c_{n+p} z^{n+p}\right| \\
& -\left|2(p-\gamma) z^{p}+\sum_{n=1}^{\infty}(n+p) a_{n+p} z^{n+p}+(p-2 \gamma) \sum_{n=1}^{\infty} c_{n+p} z^{n+p}\right| \\
\leq \sum_{n=1}^{\infty}|(n+p)| a_{n+p}|z|^{n+p}+p \sum_{n=1}^{\infty}\left|c_{n+p}\right||z|^{n+p} & \\
& -\left[\begin{array}{c}
\left.2(p-\gamma)|z|^{p}-\sum_{n=1}^{\infty}(n+p)\left|a_{n+p}\right||z|^{n+p}-\right] \\
(|p-2 \gamma|+p) \sum_{n=1}^{\infty}\left|c_{n+p}\right||z|^{n+p}
\end{array}\right] \\
\leq & {\left[\begin{array}{c}
\left.-2(p-\gamma)+\sum_{n=1}^{\infty}(n+p)\left|a_{n+p}\right|+(|p-2 \gamma|+p) \sum_{n=1}^{\infty}\left|c_{n+p}\right|\right]|z|^{p}
\end{array}\right.}
\end{aligned}
$$

therefore $\Lambda<0$ and we have

$$
\left|z f^{\prime}(z)-p \frac{g(z) g(t z)}{z^{p} t^{p}}\right|<\left|z f^{\prime}(z)-\frac{(p-2 \gamma) g(z) g(t z)}{z^{p} t^{p}}\right|
$$

which is equivalent to (1.3) and also to the inequality (1.2). Thus and it completes the proof.

Theorem 2.5 If $f \in \chi_{p}^{t}(\gamma)$ then we have

$$
\frac{1-(p-2 \gamma) r}{(1+r)^{3}} \leq\left|f^{\prime}(z)\right| \leq \frac{1+(p-2 \gamma) r}{(1+r)^{3}} \quad(|z|=r, 0 \leq r<1)
$$

and

$$
\int_{0}^{r} \frac{1-(p-2 \gamma) \tau}{(1+\tau)^{3}} d \tau \leq|f(z)| \leq \int_{0}^{r} \frac{1+(p-2 \gamma) \tau}{(1+\tau)^{3}} d \tau \quad(|z|=r, 0 \leq r<1)
$$


These results are sharp.

Proof. Suppose that $f \in \chi_{p}^{t}(\gamma)$. From the definition of subordination between analytic functions, we deduce that

$$
\begin{gathered}
\frac{1-(p-2 \gamma) r}{(1+r)} \leq \frac{1-(p-2 \gamma)|w(z)|}{(1+|w(z)|)} \leq\left|\frac{t^{p} z^{p+1} f^{\prime}(z)}{g(z) g(t z)}\right|=\left|\frac{z f^{\prime}(z)}{F(z)}\right| \leq \\
\frac{1+(p-2 \gamma)|w(z)|}{(1+|w(z)|)} \leq \frac{1+(p-2 \gamma) r}{(1+r)}
\end{gathered}
$$

where $w$ is Schwarz function with $w(0)=0$ and $|w(z)|<1, z \in U$. Since

$$
\left[F(z)=\frac{g(z) g(t z)}{t^{p} z^{p}}(|z|=r, 0 \leq r<1)\right]
$$

is an starlike function, it is well known that [1]

$$
\frac{r}{(1+r)^{2}} \leq|F(z)| \leq \frac{r}{(1-r)^{2}} \quad(|z|=r<1) .
$$

Now it follows from (2.14) and (2.15), that

$$
\left[\frac{1-(p-2 \gamma) r}{(1+r)^{3}} \leq\left|f^{\prime}(z)\right| \leq \frac{1+(p-2 \gamma) r}{(1+r)^{3}} \quad(|z|=r, 0 \leq r<1)\right]
$$

Let $z=r e^{i \theta}(0 \leq r<1)$. If $L$ denotes that closed line segment in the complex $\zeta$ plane from $\zeta=0$ to $\zeta=z$, then we have

$$
f(z)=\int_{L} f^{\prime}(\zeta) d \zeta=\int_{0}^{r} f^{\prime}\left(\tau e^{i \theta}\right) d \tau(|z|=r, 0 \leq r<1)
$$

Thus by using upper estimate in (2.12), we have

$$
|f(z)|=\left|\int_{0}^{z} f^{\prime}(\zeta) d \zeta\right| \leq \int_{0}^{r} f^{\prime}\left(\tau e^{i \theta}\right) d \tau \leq \frac{1+(p-2 \gamma) \tau}{(1+\tau)^{3}} d \tau \quad(|z|=r, 0 \leq r<1)
$$

which yields the right hand side of the inequality in (2.13). In order to prove the lower bound in (2.13), it is sufficient to show that it holds true for $z_{0}$ nearest to zero, where $\left|z_{0}\right|=r(0<r<1)$. Moreover, we have

$$
|f(z)| \geq\left|f\left(z_{0}\right)\right|(|z|=r, 0 \leq r<1)
$$

Since $\mathrm{f}(\mathrm{z})$ is close-to-convex function in the open unit disk $U$, it is univalent in $U$. We deduce that the original image of the closed line segment $L_{0}$ in the complex $\zeta$ plane from $\zeta=0$ to $\zeta=z_{0}$ is a piece of arc $\Gamma$ in the disk $U_{r}$, given by

$$
U_{r}=\{z: z \in C \text { and }|z|=r, 0 \leq r<1\}
$$

Since, in accordance with (2.12), we have

$$
|f(z)|=\int_{f(\Gamma)}|d(w)|=\int_{\Gamma}\left|f^{\prime}(z)\right||d z| \geq \int_{0}^{r} \frac{1+(p-2 \gamma) \tau}{(1+\tau)^{3}} d \tau(|z|=r, 0 \leq r<1)
$$


This completes the proof of Theorem 2.5.

In order to give the inclusion relationship for the class $\chi_{p}^{t}(\gamma)$, we shall require the following lemma.

lemma 1. let $-1 \leq B_{2} \leq B_{1}<A_{1} \leq A_{2} \leq 1$. Then

$$
\frac{1+A_{1}(z)}{1+B_{1}(z)} \prec \frac{1+A_{2}(z)}{1+B_{2}(z)}
$$

Theorem 2.6 let $0 \leq \gamma_{1} \leq \gamma_{2}<p$.

then we have $\chi_{p}^{t}\left(\gamma_{1}\right) \subset \chi_{p}^{t}\left(\gamma_{2}\right)$

Proof Suppose that $f \in \chi_{p}^{t}\left(\gamma_{1}\right)$, by theorem 1 we have

$\frac{z f^{\prime}(z)}{g(z)} \prec \frac{1-\left(p-2 \gamma_{1}\right) z}{(1-z)}$

since $0 \leq \gamma_{1} \leq \gamma_{2}$, then we have

$-1 \leq p-2 \gamma_{2} \leq p-2 \gamma_{1}<1$

thus by lemma 1 , we have

$\frac{z f^{\prime}(z)}{g(z)} \prec \frac{1-\left(p-2 \gamma_{2}\right) z}{(1-z)}$

that is $f \in \chi_{p}^{t}\left(\gamma_{2}\right)$, This means $\chi_{p}^{t}\left(\gamma_{1}\right) \subset \chi_{p}^{t}\left(\gamma_{2}\right)$. Hence the proof is complete.

\section{REFERENCES}

I. Graham and G. Kohr, Geometric Function Theory in One and Higher Dimensions, Marcel Dekker, Inc. (2003).

C. Geo, S. Zhou, On a class of analytic functions related to the class to the starlike functions, Kyungpook Math. J. 45(2005), 123-130.

J. Kowalczyk and E. Le's-Bomba, On a subclass of close-to-convex functions, Appl. Math. Letters 23(2010), 1147-1151.

Qing-Hua Xu, H. M. Srivastava and Zhou Li, A certain subclass of analytic and close-to-convex functions, Appl. Math. Letters 24(2011), 396-401.

Zhi-Gang Wang and Da-Zhao Chen, On a certain subclass of close-to-convex functions, Hacet. J. Math. Stat. 38(2)(2009), 95-101.

\section{I.B. Bapana,}

Department of Mathematics, Slovak Technical University,

Department of Mathematics, Govt. Postgraduate College,

Bhilwara-311001, Rajasthan,

India

E-mail: bapnain@yahoo.com

Sneha Handwana,

Department of Mathematics, Slovak Technical University,

Department of Mathematics, Govt. Postgraduate College,

Bhilwara-311001, Rajasthan,

India

E-mail: bapnain@yahoo.com

Received September 2013 